The immunotherapy is currently changing the landscape of oncology. Nowadays the standard of care in metastatic or unresectable melanoma patients include immunomodulating modalities such as anti-PD-1 drugs (nivolumab, pembrolizumab) and anti-CTLA-4 antibody ipilimumab. The improvements of progression free survival and overall survival connected with those treatments were unprecedented and have been confirmed in stage III trials. The efficacy of immunotherapy in metastatic setting can be further upgraded in some groups of patients by combining both types of antibodies. Latest clinical data suggest that treatment with immunotherapy can be also favorable for patients in adjuvant setting. Other treatment approaches based on immunological response (e.g. oncolytic viruses or adoptive cell therapy) have been proven useful in specific clinical situations. The future of melanoma treatment is still evolving, new molecular targets are being invented and hopefully current endeavors will led to further improvement of patients' survival. This review aims to summarize current state of immunotherapy in melanoma and identifying possible directions of development.

Key words: melanoma, nivolumab, pembrolizumab, ipilimumab.

Contemp Oncol (Pozn) 2018; 22 (1A): 61-67 DOI: https://doi.org/10.5114/wo.2018.73889

\section{Immunotherapy of melanoma}

\author{
Iwona Lugowska, Pawel Teterycz, Piotr Rutkowski
}

Department of Soft Tissue/Bone Sarcoma and Melanoma, Maria Sklodowska-Curie Institute - Oncology Center, Warsaw, Poland

\section{Introduction}

The immunotherapy is currently changing the landscape of oncology. The molecular mechanism of immunotherapy is based on the interaction between immune system and molecules present on the surface of cancer cell. The immune response against neoplasms is mainly mediated by the adaptive immune system and cytotoxic lymphocytes T. In order to react against any foreign cell, naïve lymphocytes T must be activated and thus cytotoxic response can be started via two signals: the first signal is mediated by $T$ cell receptor (TCR) connected to a specific antigen on an antigen presenting cell (APC), and the secondary signal is based on the interaction between a CD28 receptor on lymphocyte surface and CD80/86 on APC. Immune cells can also receive inhibitory signals in order protect from autoimmunological reactions, to reduce inflammation, and to enable fetomaternal tolerance. So far, many antibodies has been approved for cancer treatment (e.g. rituximab, trastuzumab, alemtuzumab, avelumab).

The first agents of FDA-approved immunotherapies in metastatic/unresectable cutaneous melanomas, which are accountable for the vast majority of deaths caused by skin cancers, include anti-PD-1 drugs (nivolumab, pembrolizumab) and anti-CTLA-4 antibody ipilimumab. In Poland those therapies are available as the first or second line settings of treatment of advanced melanomas. The future of melanoma treatment is still evolving and hopefully will led to further improvement of patients' survival. The results presented during the last American Society of Clinical Oncology (ASCO) Meeting 2017 showed a high rate of responses on further combinations of immune checkpoint inhibitors (anti-CTLA4 and anti-PD1) with different types of immunotherapies, or targeted therapies. Nowadays, numerous of clinical trials actively explore different combinations especially for melanoma patients who had progression on immune checkpoint inhibitors. Addition of epacadostat to pembrolizumab proved to be safe and effective (response rate $56 \%$, with $6 \%$ complete remissions), but dependent on the presence of the protein PD-L1 in tumors (71\% response rate in PD-L1-positive patients versus 29\% in PD-L1-negative patients). The last data coming from clinical trials dedicated to patient who experienced disease progression on immunotherapies proved the safety and efficacy of the immune checkpoint protein LAG-3 antibody in combination with nivolumab which was assessed in 55 melanoma patients. The response rate reached $12.5 \%$ in this heavily pre-treated cohort. The LAG-3 expression in tumor tissue was a predictive marker - in LAG-3 positive patients the response was significantly higher than in patients with a LAG-3 presence of less than $1 \%$ of positive tumor cells, the response rate equaled a $20 \% \mathrm{vs} .7 \%$ [1]. The next combination of an oncolytic cold virus (CVA21) with ipilimumab tested in 22 patients confirmed a high rate of long duration responses in 10 patients (4 patients had complete remission). The promising opportunity in melanoma patients previously treated with immune checkpoint antibodies is entinostat with pembrolizumab due to a $31 \%$ response rate.

The first indications which was proven to be beneficial for patients were renal cancer and melanoma [2, 3]. High-dose bolus IL-2 (HD IL-2) was approved by the FDA in 1998 for the treatment of metastatic melanoma due to the potential 
for durable complete responses. The published overall response rate (ORR) was 16\% (CR 6\%, PR 10\%) and 60\% of the complete responders had durable responses, thus suggesting the potential for long-term control of micrometastatic disease by HD IL-2. The administration of HD IL-2 requires hospitalization with intensive monitoring and is mostly limited to specialized centers with personnel who is experienced in the management of this regimen. During this treatment patients are at risk of multiorgan complications, so often only younger patients with excellent performance status were eligible [4, 5]. The second drug approved by the FDA, but only for adjuvant therapy of resected high-risk melanoma, was interferon $\alpha$ (IFN- $\alpha$, Intron A). In patients with metastatic melanoma, INF- $\alpha$ was associated with antitumor activity at ORR 22\% (CR $<4 \%$ ) but responses were limited to patients with low-tumor load mainly in cutaneous or soft-tissue sites [6]. The quality of life was significantly affected by side effects such as fever, fatigue, myalgia, and autoimmune events. Pegylated IFN- $\alpha$ (PEG-Intron) permits good tolerability with similar efficacy in metastatic disease [7]. The clinical benefit after HD IL-2 and/ or INF- $\alpha$ has provided "proof of concept" for further research in the field of immunooncology and allowed immunotherapy to intensively develop in the last decade.

\section{Checkpoint inhibitors}

\section{Antibody against CTLA-4}

In 1987, James P. Allison identified cytotoxic T-lymphocyte antigen 4 (CTLA-4) preventing T cells from attacking tumor cells. The hypothesis was that blockage of the CTLA4 would allow to active the immune system against cancer cell. In 1996, there were the first data about antitumor activity of blockade of CTLA-4 molecules in mice, which enable to introduce the new molecule into the early phase of clinical trial. In 2010, the outstanding data from the III phase MDX010-20 study about the antitumor activity of ipilimumab were presented, and immediately afterward the drug was registered in advanced melanoma. Its efficacy as 1-year and 2-year survival rates were equal to $46 \%$ and $24 \%$ for ipilimumab and $25 \%$ and $14 \%$ for control group respectively. The most extensive group was described in a paper by Schadendorf et al. and included 1861 cases from 2 III phase, 8 II phase trials, 2 observational studies as well as 2965 patients who received ipilimumab in an expanded access program. The follow-up period reached 10 years. The 3-year survival rate was equal to $21 \%$ and after this period plateau was observed with the safety issue related to the effect of activation of the autoreactive lymphocytes, which occurred in $18-23 \%$ of the patients. The most common ones included colitis, skin lesions and endocrinopathies [8, 9].

\section{Antibody against programmed death-1 (PD-1)}

In the 1990s, Okazaki et al. discovered a molecule on T cells, which was called programmed death 1 (PD-1) [10]. Almost 20 years later, an antibody that targeted PD-1 was developed. The clinical data indicates that anti-PD1 immunoglobulins have been the most effective in melanoma. In the CheckMate 066, such drug (nivolumab) was given to 210 treatment-naive patients with unresectable or metastatic melanoma. The median OS was not reached and the 1-year survival rate was as high as $72.9 \%$. The median PFS was equal to 5.1 months [11]. The KEYNOTE-006 trial was comparing pembrolizumab administrated every 2 weeks or every 3 weeks to ipilimumab in 4 doses every 3 weeks. The 1 -year survival rates reached $74.1 \%, 68.4 \%$ and $58.2 \%$ in each group respectively [12]. Both nivolumab and pembrolizumab showed unprecedented efficacy and greatly improved prognosis in melanoma patients. Latest data also suggested that the response to treatment is durable even after ending the immunotherapy. Long term results of KEYNOTE-006 trial shows that $91 \%$ of patients who ended 2-year long protocol did not progress [13]. Both nivolumab and pembrolizumab show even more preferable safety profile than ipilimumab. The immune-related adverse events in grade 3 or 4 (according to the CTCAEv4) were present in 10.1-13.3\% of patients and were usually manageable with the use of systemic glucocorticosteroids.

Clinical data confirms that immune checkpoint inhibitors showed especially good activity among patients treated for tumors such as melanoma, non-small cell lung carcinoma or bladder cancer. It has been theorized that the response during immunotherapy depends on the number of so called neoantigens - antigens presented on tumor cells which are effect of tumor-specific mutations [14, 15]. Indeed, neoplasms mentioned above are characterized by an especially large number of somatic mutations (mutational tumor load) [16]. Furthermore, some data suggests that even among melanoma tumors, there is a correlation between mutational tumor load and degree of clinical benefit from immunotherapy [17].

\section{Combination of anti-PD-1 and anti-CTLA-4}

Based on the results of the preclinical studies it has been proven that the inhibition of two checkpoint inhibitors (anti PD-1 and anti CTLA-4) was associated with the increased infiltration of inflammatory cells ( $T$ lymphocytes) and significantly higher antitumor efficacy of combination therapy than ipilimumab alone. The results from the Checkmate-069 and -067 studies establish that the combination produces impressive clinical antitumor activity and suggests that all of the antitumor effects of immunotherapy are not subsumed in the activity of single agent PD-1 blockade. In 2015 year the results of the phase III study CA209-067 (CheckMate 067) addressed to the metastatic/unresectable melanoma population, confirmed the clinical benefit of combination of nivolumab and ipilimumab over the monotherapy with checkpoint inhibitors [18]. The highest response rate was achieved in the combination arm, but a monotherapy with nivolumab was superior to ipilimumab alone; the responses were $57.6,43.7$ and $19 \%$, respectively. What is more, the combination of checkpoint inhibitors efficacy was the highest published so far in metastatic melanoma patients - median PFS was 11.5 months (95\% Cl: 8.9-16.7), and was significantly longer than median on ipilimumab alone - 2.9 months $(95 \% \mathrm{Cl}: 2,8-3,4)$. The overall survival were also improved on combination therapy (2-year OS was 64\% for combination vs. 59\% for monotherapy with nivolum$\mathrm{ab}$, and $45 \%$ - for ipilimumab). Treatment-related adverse 
events of any grade occurred in $82.1 \%$ of the patients in the nivolumab group, $95.5 \%$ of those in the nivolumab-plus-ipilimumab group, and $86.2 \%$ of those in the ipilimumab group. The most common adverse events in the combination arm were diarrhea (44.1\%), fatigue (35.1\%), and pruritus (33.2\%). The grade $3 / 4$ of adverse events were also higher in the nivolumab and ipilimumab group (55.0\%) than in the nivolumab group (16.3\%) or the ipilimumab group (27.3\%). The presented results confirm the clinical utility of the combination therapy over monotherapy but it is obligatory to bear in mind the toxicity profile. In the biomarkers study, patients were stratified according the PD-L1 status, and in population with a positive PD-L1 (higher that $5 \%$ of cells with positive expression), the median progression-free survival was 14.0 months $(95 \% \mathrm{Cl}$ : 9.1 to not reached) in the nivolumab group, 14.0 months ( $95 \% \mathrm{Cl}: 9.7$ to not reached) in the nivolumab-plus-ipilimumab group, and 3.9 months (95\% Cl: 2.8-4.2) in the ipilimumab group. Among patients with a negative PD-L1 tumor status, the median progression-free survival was 5.3 months (95\% Cl: 2.8-7.1), 11.2 months (95\% Cl: 8.0 to not reached), and 2.8 months ( $95 \% \mathrm{Cl}: 2.8-3.1)$, respectively. Concluding, the combination of checkpoint inhibitors is the best option when the PD-1 expression is below 5\%, in other cases - the nivolumab alone is sufficient to reach the satisfactory results in terms of survival with less toxic profile. Nowadays ipilimumab is not considered as a first line treatment in metastatic melanoma, and the nivolumab or pembrolizumab should be administered as monotherapy or combination with anti-CTLA-4 antibodies [19].

In the light of the latest research, the combination of therapy is mostly required in the patients with brain metastases (the population with very unfavorable prognosis). In 2017, the published data of the CheckMate 204 study showed the significant improvement of survival of patients with asymptomatic brain metastases (at least one metastases of the size: $0.5-3 \mathrm{~cm}$ ) receiving the combination therapy over the monotherapy with ipilimumab. The treatment was based on the following schema: nivolumab $(1 \mathrm{mg} / \mathrm{kg})$ and ipilimumab (3 mg/kg every 3 weeks $\times 4)$ followed by nivolumab $3 \mathrm{mg} / \mathrm{kg}$ every 2 weeks until disease progression or unacceptable toxicity. The stereotactic radiosurgery was allowed in this study. Due to the $56 \%$ of intracranial responses reached in this study, it is suggested that combination may be a new treatment paradigm in patients with asymptomatic brain metastases [20]. The studies dedicated to the patients with symptomatic brain metastases showed also promising results of combination of checkpoint inhibitors. The results of randomized phase II study, where patients were assigned to the one of two cohorts: nivolumab with ipilimumab or nivolumab with placebo, indicate that the intracranial response also was significantly better on combination (ORR was 44\%) than in monotherapy (ORR was 20\%); the 6-month progression free survival was 50\% [21].

\section{Talimogene laherparepvec (T-VEC)}

Whereas previously described substances are monoclonal antibodies and work in the mechanism of checkpoint blockage, the last one - T-VEC is a unique example of an oncolytic virus which was genetically modified to target tumor cells as well as to boost antineoplastic immune response [22]. During the genetic engineering of T-VEC, the gene for granulocyte macrophage colony-stimulating factor (GM-CSF) was induced while genes encoding ICP34.5 and ICP47 have been completely deleted in order to ensure tumor selectivity [23]. In a phase III study, T-VEC was compared with subcutaneous injections of GM-CSF on a group of 436 patients with stage IIIB, IIIC or IV unresectable melanoma. It showed noticeable clinical benefit with durable responses in the subgroup of patients with stage IIIB/IIIC/ IVA disease where the median OS reached 41.1 months [24]. The summary of the use and results of therapy with immunomodulating agents used currently in melanoma therapy is presented in Table 1.

\section{Adoptive cell therapy}

Adoptive cell therapy (ACT) involves collection of lymphocytes from the patient, followed by in vitro selection, expansion and their activation with subsequent infusion of processed lymphocytes back into the patient to induce an immune anticancer responses. The cells most commonly used for ACT are peripheral blood lymphocytes or tumor-infiltrating lymphocytes [TIL]. A recent reports demonstrates an ORR of $56 \%$ observed in 93 patients with manageable toxicities. Patients, who achieved a complete response, has durable relapse free survival with a median follow-up of 31 months [25]. A novel approach of ACT is the infusion of isolated and expanded autologous CD4+ T-cell activated by the melanoma-associated antigen - NYESO-1. In patients with refractory metastatic melanoma, the transferred CD4+ T cells also mediated a durable remission, additionally against melanoma antigens other than NY-ESO-1 [26]. These complex therapies require the development of a "custom made drug" for each patient, with weeks of cell culture, skilled man-hours, and patient preparation. The hurdles that must be surpassed for ACT are economic costs, and their availability limited only to specialized and subsidized centers experienced with immunotherapies and toxicity management. What is more, immune cells can be genetically engineered to express a tumor-specific receptor, cultured and returned to the patient. Natural killer cells, lymphokine-activated killer cells, cytotoxic T cells and dendritic cells are used the most commonly in this procedure. The cost of this treatment is high, and the only one approved cell-based therapy is Sipuleucel-T registered for the treatment of prostate cancer.

\section{Current standard of care of advanced melanoma}

\section{Treatment of metastatic melanoma}

Modern systemic treatment of unresectable or metastatic melanoma includes targeted therapy and immune- therapeutics. Targeted therapy has been developed for melanoma with the hot-spot mutation in BRAF codon V600 which is present in about $50 \%$ of cases [11]. It has been proven in a number of studies that the most effective treatment for this group of patients consists of the combination of a BRAF inhibitor and MEK inhibitor [12-14]. IO agents approved globally for routine clinical 
Table 1. Summary of the use and results of therapy with immunomodulating agents available in melanoma

\begin{tabular}{|c|c|c|c|}
\hline Drug name & Registration & Efficacy in melanoma & Side effects \\
\hline $\begin{array}{l}\text { Ipilimumab } \\
\text { (anti-CTLA-4) }\end{array}$ & $\begin{array}{l}\text { Advanced melanoma (unresectable or } \\
\text { metastatic) in adults, adjuvant therapy in } \\
\text { stage III (in US) }\end{array}$ & $\begin{array}{l}\text { Median OS: } \\
10.1 \text { months (pretreated patients) } \\
11.2 \text { (first line) } \\
\text { ORR: } \\
\text { 11\% (pretreated patients) } \\
\text { 15\% (first line) } \\
2 \text {-year survival rate: } \\
24 \% \text { (second line) } \\
28.5 \% \text { (first line) } \\
\text { Noted } 10 \text {-year survival rate }-20 \% \text { (plateau) }\end{array}$ & $\begin{array}{l}\text { Adverse effects of 3-4 } \\
\text { grade in } 15-56 \% \text { of cases } \\
\text { (abdominal pain, severe } \\
\text { diarrhoea or significant } \\
\text { change in the number } \\
\text { of stools, blood in stool, } \\
\text { Severe elevations in LFT, } \\
\text { life threatening skin rash, } \\
\text { nephritis, pneumonitis, } \\
\text { pancreatitis, non- } \\
\text { infectious myocarditis) }\end{array}$ \\
\hline $\begin{array}{l}\text { Nivolumab } \\
\text { (anti-PD-1) }\end{array}$ & $\begin{array}{l}\text { Advanced melanoma (unresectable or } \\
\text { metastatic) in adults as monotherapy or } \\
\text { in combination with ipilimumab (only in } \\
\text { patients with low tumor PD-L1 } \\
\text { expression) } \\
\text { Non-small cell lung cancer } \\
\text { Renal cell carcinoma } \\
\text { Classical Hodgkin lymphoma } \\
\text { Squamous cell cancer of the head and } \\
\text { neck } \\
\text { urothelial carcinoma }\end{array}$ & $\begin{array}{l}\text { ORR: } 40-41 \% \text { ( } 60 \% \text { in combination with } \\
\text { ipilimumab) } \\
\text { 2-year survival rate: } 57.7 \% \text { ( } 64 \% \text { in } \\
\text { combination with ipilimumab) } \\
\text { 3-year survival rate: } 52 \% \text { ( } 58 \% \text { in } \\
\text { combination with ipilimumab) }\end{array}$ & $\begin{array}{l}\text { Adverse effects of } \\
3-4 \text { grade in } 12-14 \% \\
\text { of patients (mainly } \\
\text { autoimmune response). } \\
\text { In combination with } \\
\text { ipilimumab grade } 3-4 \\
\text { side effects occur in } \\
54-55 \%\end{array}$ \\
\hline $\begin{array}{l}\text { Pembrolizumab } \\
\text { (anti-PD-1) }\end{array}$ & $\begin{array}{l}\text { Advanced melanoma (unresectable or } \\
\text { metastatic) in adults } \\
\text { Non-small cell lung cancer } \\
\text { classical Hodgkin lymphoma }\end{array}$ & $\begin{array}{l}\text { ORR: } 33-45 \% \\
\text { 2-year survival rate: } 55 \%\end{array}$ & $\begin{array}{l}\text { Adverse effects of } \\
3-4 \text { grade in } 13-18 \% \\
\text { of patients (mainly } \\
\text { autoimmune response) }\end{array}$ \\
\hline $\begin{array}{l}\text { T-VEC } \\
\text { (talimogene } \\
\text { laherparepvec) }\end{array}$ & $\begin{array}{l}\text { Local treatment of melanoma with } \\
\text { unresectable metastases to skin, } \\
\text { subcutaneous tissue and lymph nodes }\end{array}$ & $\begin{array}{l}\text { Median OS: } 23.3 \text { months } \\
\text { ORR: } 26.4 \%\end{array}$ & Mainly flu-like symptoms \\
\hline
\end{tabular}

usage include three checkpoint inhibitors - two anti-PD1 antibodies (nivolumab and pembrolizumab), one anti-CLTA-4 immunoglobulin (ipilimumab) and a representative of a new therapeutic group, oncolytic virus, talimogene laherparepvec (T-VEC).

\section{Adjuvant therapy}

Until recently, the standard of care for resected melanoma was treatment with interferon. The implication of this treatment on survival were limited. However the published results from the EORTC 18071 trial have changed this standard of care. This phase 3 randomized trial compared ipilimumab $(10 \mathrm{mg} / \mathrm{kg})$ to placebo in patients who had undergone complete resection of stage III melanoma. In this study, 951 patients were randomly (1: 1) assigned to treatment with ipilimumab every 3 weeks for 4 doses, then every 3 months for up to 3 years or until disease recurrence or to placebo. The 5-year rate of recurrence-free survival was $41 \%$ in the ipilimumab group and $30 \%$ in the placebo group. The 5 -year overall survival was $65 \%$ in the ipilimumab group and 54\% in the placebo group. With 2.7 median follow up, the median relapse-free survival (RFS) on ipilimumab arm was significantly superior that in placebo; 26.1 vs. 17.1 months respectively. The improvement was noticed in both populations: with macro- and/or micrometastases to the regional lymph nodes. The effect of treatment was even more remarkable in population with ulceration of primary site. The treatment related side ef- fects were significantly more commonly observed in ipilimumab group than in placebo: $54 \%$ patients experienced $3 / 4$ grade of toxicity acc. CTCEA v. 4 in comparison to $25 \%$ of the placebo group. What is more, in half of population treatment with ipilimumab, the therapy must have been stopped due to the safety issue, 5 patients (1\%) died because of ipilimumab toxicity [27]. In 2015 this adjuvant ipilimumab gained US Food and Drug Administration (FDA) approval, but practically this treatment has limited usage due to the high rated of toxicity and cost (about 1 million US dollar per patient). On the other hand, the presented in 2016 update of trial (with 5.3 median follow up), indicates the significant improvement of RFS, and overall survival (OS). The 5 -year OS in ipilimumab group was $65,4 \%$, and was significantly higher than in placebo group - 54,4\%; (HR 0,72, 95,1\% Cl: 0,58-0,88; $p=0,001$ ) [28]. Nowadays the clinical trials are ongoing with anti-PD1 antibodies in adjuvant settings. The first data presented in 2017 showed, that nivolumab administered in patients with melanoma after lymphadenectomy (IIIB, IIIC and IV Stage) is related to the $10 \%$ of RFS improvement with lower toxicity as compared with ipilimumab [29]. The trial with combination anti-CTLA-4 and anti-PD-1 are ongoing, as well, the results are pending.

\section{Future directions}

As mentioned above, currently first line therapeutic options for advanced melanoma include immunotherapy with 
anti-PD1 antibodies (combination of PD1/CTLA-4 blockers can be an option in a specific group of patients) or targeted therapy with BRAF and MEK inhibitors in BRAF V600 mutated cases whereas dacarbazine-based chemotherapy should be considered only in later lines of treatment [30, 31]. Yet the data on the optimal first line treatment (IO or targeted therapy) are sparse. New therapeutic agents for immunomodulatory treatment are also emerging rapidly. There is research concerning fully utilizing available treatment options as well as developing new drugs.

The best first line treatment in the whole population of advanced melanoma patients is unknown. Some small academic clinical trials try to answer this question in specific subgroups of cases. For example, an Australian trial, Anti-PD1 Brain Collaboration (ABC) focused on 76 melanoma patients with brain metastases. Patients received nivolumab or nivolumab and ipilimumab. It has been shown that combination of these two drugs was more effective with an objective response rate of $42 \%$ months whereas nivolumab monotherapy resulted in $20 \%$ or $6 \%$ ORR (in asymptomatic and neurologically symptomatic patients respectively). The activity of nivolumab +ipilimumab was high only when it was the first line treatment - after BRAFi/MEKi therapy ORR was only 16\% (NCT02374242) [21]. On the other hand in the study by Schreuer et al patients who progressed on targeted therapy and subsequently received immunotherapy were re-challenged with a combination of dabrafenib and trametinib and in 18/25 (72\%) cases clinical benefit was noted which may suggest preferable start with targeted therapy in order to maximize the survival outcome [32].

A thrilling therapeutic option is a combination of simultaneous PD1/PD-L1 blockage and inhibition of BRAF pathway. There are currently ongoing trial that facilitate treatment with pembrolizumab, dabrafenib and trametinib (NCT02130466) and atezolizumab (anti-PD-L1) with vemurafenib and cobimetinib (NCT02908672).

There are several immunomodulatory receptors both on the surface of lymphocytes and in the tumor microenvironment which can serve as potential targets in enhancing the antineoplastic cytotoxic effect of the immune system. Some of them are summarized in Table 2. Arguably the next most attractive target in 10 management of melanoma after PD1/PD-L1 pathway is indoleamine 2,3-dioxygenase (IDO). IDO is an enzyme encoded by gene IDO1 which takes part in tryptophan catabolism. It catalyzes the first, rate-limiting step of conversion of tryptophan into N'-Formylkynurenine. IDO, similarly to PD-L1 is highly expressed in the human placenta, but it was also detected in malignant tumor microenvironment [33-36]. The preclinical data suggest that tryptophan depletion induced by IDO results in inhibition $T$ cell proliferation and promotion of Treg activity $[37,38]$. Epacadostat is the first selective, oral IDO inhibitor subject to clinical trials. In a phase I study it showed an acceptable toxicity profile and clinical benefit for 7 out of 52 cases was noticed. Currently a phase III

Table 2. Some of potential molecular targets in immunomodulatory therapy

\begin{tabular}{|c|c|c|}
\hline Receptor & Description & Example of drug \\
\hline PD-L1 & $\begin{array}{l}\text { It is main ligand to PD1 receptor present on T lymphocytes. Their interaction leads to inhibition of } 1 \mathrm{l}-2 \\
\text { production and T cell proliferation [40]programmed death-1 (PD-1 }\end{array}$ & $\begin{array}{l}\text { Atezolizumab, } \\
\text { avelumab }\end{array}$ \\
\hline IDO1 & $\begin{array}{l}\text { IDO is short for Indoleamine 2,3-dioxygenase. This enzyme limits rate of tryptophan catabolism. It has } \\
\text { been shown that expression of IDO in APCs modulates activity of T cells in human placenta [41]. When } \\
\text { overexpressed in tumours, it promotes their immune resistance [42] }\end{array}$ & Epacadostat \\
\hline LAG3 & $\begin{array}{l}\text { LAG3 is short for Lymphocyte-activation gene } 3 \text {. This protein is similar to CD4 receptor and binds to } \\
\text { MHC II. It takes part in downregulating proliferation and activation of T cells }[1,43]\end{array}$ & $\begin{array}{l}\text { BMS-986016, } \\
\text { LAG525 }\end{array}$ \\
\hline $\mathrm{B} 7-\mathrm{H} 3$ & $\begin{array}{l}\text { B7-H3, also known as CD 276, is a member of B7 family of proteins and as such can bind to CLTA-4 } \\
\text { receptor and inhibit lymphocyte T activation [44] }\end{array}$ & Enoblituzumab \\
\hline OX40 & $\begin{array}{l}\text { is a member of the TNF receptor superfamily. It promoted T lymphocytes activity and memory as well as } \\
\text { suppress subpopulation of Treg cells }\end{array}$ & MEDI0562 \\
\hline CD122 & is a subunit of IL-2 receptor & NKTR-214 \\
\hline CD40 & $\begin{array}{l}\text { is a receptor on antigen presenting cells. It interacts with CD4OL on Th cells. Its stimulation leads to } \\
\text { increased level of activity in APCs such as macrophages and B lymphocytes }\end{array}$ & APX005M \\
\hline GITR & $\begin{array}{l}\text { is a member of the TNF receptor superfamily. This protein is presented on T lymphocytes and acts } \\
\text { as co-stimulat to TCR signal. It has been showed that activating GITR promotes T cell (including Treg) } \\
\text { proliferation and promotes loss of suppressive activity of Treg cells [45]including T lymphocytes. } \\
\text { Because of a high homology in its cytoplasmic region with other known costimulatory members of the } \\
\text { TNFRSF, we investigated whether GITR played a costimulatory role in T lymphocyte subpopulations. Our } \\
\text { results show that the proliferation response of CD8+ and CD4+ peripheral T cell subpopulations was } \\
\text { potentiated when a GITR costimulus was added to an anti-CD3 stimulus. Furthermore, expression of the } \\
\text { main activation-induced receptor (IL-2Ralpha }\end{array}$ & TRX518 \\
\hline TIM-3 & $\begin{array}{l}\text { T-cell immunoglobulin and mucin-domain containing-3 is also known as Hepatitis A virus cellular } \\
\text { receptor } 2 \text { (HAVCR2). It is an immune checkpoint receptor that limits the duration and scale of Th1 and } \\
\text { Tc responses [46] }\end{array}$ & $\begin{array}{l}\text { MBG453, TSR- } \\
022\end{array}$ \\
\hline KIR & $\begin{array}{l}\text { Killer cell immunoglobulin-like receptor is a family of NK cell negative regulator. NK cells mediate } \\
\text { spontaneous cytotoxicity and antibody-dependent cell-mediated cytotoxicity (ADCC). }\end{array}$ & Lirilumab \\
\hline
\end{tabular}


Table 3. Clinical studies on immunotherapy agents currently conducted in melanoma

\begin{tabular}{|c|c|c|}
\hline Study & Combination & Description \\
\hline NCT02967692 & PD1 inhibitor, BRAFi, MEKi & $\begin{array}{l}\text { Phase III study evaluating efficacy and safety of melanoma treatment composed } \\
\text { of novel anti-PD1 antibody and targeted therapy }\end{array}$ \\
\hline NCT02908672 & PD-L1 inhibitor, BRAFi, MEKi & $\begin{array}{l}\text { Phase III study of efficacy of atezolizumab, vemurafenib and dabrafenib } \\
\text { combination in unresectable or metastatic melanoma }\end{array}$ \\
\hline NCT02752074 & PD1, IDO1 & $\begin{array}{l}\text { Phase III study of pembrolizumab and epacadostat/placebo in advanced } \\
\text { melanoma }\end{array}$ \\
\hline NCT02705482 & $\begin{array}{l}\text { PD-L1 inhibitor/CTLA-4 } \\
\text { inhibitor, OX40 agonist }\end{array}$ & $\begin{array}{l}\text { Phase I trial evaluating safety and pharmacokinetics of novel OX40 agonist, } \\
\text { MEDI0562, in combination with durvalumab or tremelimumab (anti-PD-L1 and } \\
\text { anti-CTLA-4 respectively) in adult subjects with select advanced solid tumors }\end{array}$ \\
\hline NCT02983045 & PD1 inhibitor, CD122 & $\begin{array}{l}\text { Phase I/II study of nivolumab and novel CD122-based stimulating cytokine in } \\
\text { NSCLC, RCC and melanoma }\end{array}$ \\
\hline NCT01239134 & GITR inhibitor & $\begin{array}{l}\text { Phase } 1 \text { b study determining the safety, tolerability, pharmacokinetic of activating } \\
\text { GITR antibody, TRX518 in advanced melanoma or other solid tumors }\end{array}$ \\
\hline NCT02676869 & $\begin{array}{l}\text { PD1 inhibitor, LAG3 } \\
\text { inhibitor }\end{array}$ & $\begin{array}{l}\text { Phase I study of IMP321, novel LAG3 inhibitor combined with pembrolizumab in } \\
\text { unresectable or metastatic melanoma }\end{array}$ \\
\hline NCT02460224 & $\begin{array}{l}\text { PD1 inhibitor, LAG3 } \\
\text { inhibitor }\end{array}$ & $\begin{array}{l}\text { Phase I/II trial of safety, tolerability, pharmacokinetics (PK), pharmacodynamics } \\
\text { (PD) and anti-tumor activity of LAG525 as a single agent and in combination with } \\
\text { PDR001 (anti-PD1) in melanoma, NSCLC and RCC }\end{array}$ \\
\hline NCT02475213 & $\begin{array}{l}\text { PD1 inhibitor, B7-H3 } \\
\text { inhibitor }\end{array}$ & $\begin{array}{l}\text { Phase I study of safety of enoblituzumab in combination with pembrolizumab in } \\
\text { selected solid tumors expressing } \mathrm{B} 7-\mathrm{H} 3\end{array}$ \\
\hline NCT01714739 & $\begin{array}{l}\text { PD1 inhibitor, KIR inhibitor, } \\
\text { CTLA-4 inhibitor }\end{array}$ & $\begin{array}{l}\text { Phase I/II study assessing the safety and tolerability of lirilumab and nivolumab or } \\
\text { lirilumab and nivolumab plus ipilimumab in advanced solid tumors }\end{array}$ \\
\hline NCT02817633 & $\begin{array}{l}\text { PD1 inhibitor, TIM-3 } \\
\text { inhibitor }\end{array}$ & $\begin{array}{l}\text { Phase I, first-in-human, study evaluating safety and tolerability of the anti-TIM-3 } \\
\text { antibody TSR-022 with or without an anti-PD-1 antibody in advanced solid tumors }\end{array}$ \\
\hline NCT02608268 & $\begin{array}{l}\text { PD1 inhibitor, TIM-3 } \\
\text { inhibitor }\end{array}$ & $\begin{array}{l}\text { Phase I/II, first-in-human, study evaluating safety and tolerability of the anti-TIM-3 } \\
\text { antibody MBG } 453 \text { with or without an anti-PD-1 antibody in advanced solid tumors }\end{array}$ \\
\hline NCT02598960 & GITR agonist, PD1 inhibitor & $\begin{array}{l}\text { Phase I/II study evaluating safety and efficacy of BMS-986156 (GITR agonist) } \\
\text { Administered Alone and in Combination With Nivolumab in advanced solid tumors }\end{array}$ \\
\hline
\end{tabular}

study involving melanoma treatment with pembrolizumab +/- epacadostat is ongoing (NCT02752074). Preliminary results of a phase I/II trial showed promising 75\% (6/8) objective response rate and $100 \%(8 / 8)$ disease control rate [39].

Clinical studies on other immunomodulatory agents are summarized in Table 3.

\section{Summary}

This and many other developing melanoma immunotherapies represent the promise and potential of immunotherapy as a more effective, lifesaving cure for skin cancer.

Authors declare no conflict of interest.

\section{References}

1. Ascierto PA, Melero I, Bhatia S, et al. Initial efficacy of anti-lymphocyte activation gene-3 (anti-LAG-3; BMS-986016) in combina tion with nivolumab (nivo) in pts with melanoma (MEL) previously treated with anti-PD-1/PD-L1 therapy. J Clin Oncol 2017; 35 (15 suppl): 9520.

2. Grinspan D, Paz A, Abulafia J, Mosto S. Melanoma (Hutchinson's lentigo maligna) having spontaneous regression. A case of immunologic importance. Med Cutan Ibero Lat Am 1980; 8: 33-45.

3. Nathanson null. Spontaneous regression of malignant melanoma: a review of the literature on incidence, clinical features, and possible mechanisms. Natl Cancer Inst Monogr 1976; 44: 67-76.
4. Atkins MB, Lotze MT, Dutcher JP, et al. High-dose recombinant interleukin 2 therapy for patients with metastatic melanoma: analysis of 270 patients treated between 1985 and 1993. J Clin Oncol 1999; 17: 2105-16.

5. Atkins MB, Kunkel L, Sznol M, Rosenberg SA. High-dose recombinant interleukin-2 therapy in patients with metastatic melanoma: long-term survival update. Cancer I Sci Am 2000; 6 Suppl 1: S11-14.

6. Creagan ET, Ahmann DL, Frytak S, Long HJ, Chang MN, Itri LM. Phase II trials of recombinant leukocyte $\mathrm{A}$ interferon in disseminated malignant melanoma: results in 96 patients. Cancer Treat Rep 1986; 70: 619-24.

7. Dummer R, Garbe C, Thompson JA, et al. Randomized dose-escalation study evaluating peginterferon alfa-2a in patients with metastatic malignant melanoma. J Clin Oncol 2006; 24: 1188-94.

8. Brahmer JR, Drake CG, Wollner I et al. Phase I study of single-agent anti-programmed death-1 (MDX-1106) in refractory solid tumors: safety, clinical activity, pharmacodynamics, and immunologic correlates. J. Clin. Oncol. Off. J Am Soc Clin Oncol 2010; 28: 3167-75.

9. Schadendorf D, Hodi FS, Robert C, et al. Pooled Analysis of LongTerm Survival Data From Phase II and Phase III Trials of Ipilimumab in Unresectable or Metastatic Melanoma. J Clin Oncol 2015; 33: 1889-94.

10. Okazaki T, Honjo T. PD-1 and PD-1 ligands: from discovery to clinical application. Int Immunol 2007; 19: 813-24.

11. Robert C, Long GV, Brady B, et al. Nivolumab in previously untreated melanoma without BRAF mutation. N Engl J Med 2015; 372: 320-30.

12. Robert C, Schachter J, Long GV, et al. Pembrolizumab versus I pilimumab in Advanced Melanoma. N Engl J Med 2015; 372: 2521-32.

13. Long-term outcomes in patients (pts) with ipilimumab (ipi)-naive advanced melanoma in the phase 3 KEYNOTE-006 study who completed pembrolizumab (pembro) treatment. Abstract 9504. ASCO 2017. [index.html]. 
14. Schumacher TN, Schreiber RD. Neoantigens in cancer immunotherapy. Science 2015; 348: 69-74.

15. Le DT, Durham JN, Smith KN, et al. Mismatch-repair deficiency predicts response of solid tumors to PD-1 blockade. Science 2017; 357: 409-13.

16. Lawrence MS, Stojanov P, Polak P, et al. Mutational heterogeneity in cancer and the search for new cancer-associated genes. Nature 2013; 499: 214-8.

17. Snyder A, Makarov V, Merghoub T, et al. Genetic Basis for Clinical Response to CTLA-4 Blockade in Melanoma. N Engl J Med 2014; 371: 2189-99

18. Larkin J, Chiarion-Sileni V, Gonzalez R, et al. Combined Nivolumab and Ipilimumab or Monotherapy in Untreated Melanoma. N Engl J.Med 2015; 373: 23-34.

19. Larkin J, Chiarion-Sileni V, Gonzalez R, et al. Abstract CT075: Overall survival (OS) results from a phase III trial of nivolumab (NIVO) combined with ipilimumab (IPI) in treatment-naïve patients with advanced melanoma (CheckMate 067). Cancer Res 2017; 77 (13 Suppl): CT075-CT075.

20. Tawbi HA, Forsyth P, Algazi A, et al. Efficacy and safety of nivolum ab (NIVO) plus ipilimumab (IPI) in patients with melanoma (MEL) metastatic to the brain: Results of the phase II study CheckMate 204. J Clin Oncol 2017; 35 Suppl: abstr 9507.

21. Long GV, Atkinson V, Menzies AM, et al. A randomized phase II study of nivolumab or nivolumab combined with ipilimumab in patients (pts) with melanoma brain metastases (mets): The Anti-PD1 Brain Collaboration (ABC). Abstract 9508. ASCO 2017. ASCO 2017 Meet. Abstr.

22. Greig SL. Talimogene Laherparepvec: First Global Approval. Drugs 2016; 76: 147-54

23. Hu JCC, Coffin RS, Davis CJ, et al. A phase I study of OncoVEX GM-CSF, a second-generation oncolytic herpes simplex virus expressing granulocyte macrophage colony-stimulating factor. Clin Cancer Res 2006; 12: 6737-47.

24. Andtbacka RHI, Kaufman HL, Collichio F, et al. Talimogene Laherparepvec Improves Durable Response Rate in Patients With Advanced Melanoma. J Clin Oncol 2015; 33: 2780-8.

25. Dudley ME, Yang JC, Sherry R, et al. Adoptive cell therapy for patients with metastatic melanoma: evaluation of intensive myeloablative chemoradiation preparative regimens. J Clin Oncol 2008; 26: 5233-9.

26. Hunder NN, Wallen H, Cao J, et al. Treatment of Metastatic Melanoma with Autologous CD4+ T Cells against NY-ESO-1. N Engl J Med 2008; 358: 2698-703.

27. Eggermont AM, Chiarion-Sileni V, Grob JJ, et al. Adjuvant ipilimumab versus placebo after complete resection of high-risk stage III melanoma (EORTC 18071): a randomised, double-blind, phase 3 trial. Lancet Oncol 2015; 16: 522-30.

28. Eggermont AM, Chiarion-Sileni V, Grob JJ, et al. Prolonged Survival in Stage III Melanoma with Ipilimumab Adjuvant Therapy. N Engl J Med 2016; 375: 1845-55.

29. Weber J, Mandala M, Del Vecchio M, et al. Adjuvant Nivolumab versus Ipilimumab in Resected Stage III or IV Melanoma. N Engl J Med 2017.

30. Dummer R, Hauschild A, Lindenblatt N, et al. Cutaneous melanoma: ESMO Clinical Practice Guidelines for diagnosis, treatment and follow-up. Ann Oncol 2015; 26 Suppl 5: v126-132.

31. NCCN Guidelines Version 1.2017 Melanoma.

32. Schreuer M, Jansen Y, Planken S, et al. Combination of dabrafenib plus trametinib for BRAF and MEK inhibitor pretreated patients with advanced BRAFV600-mutant melanoma: an open-label, single arm, dual-centre, phase 2 clinical trial. Lancet Oncol 2017; 18: 464-72.

33. Su Al, Wiltshire T, Batalov S, et al. A gene atlas of the mouse and human protein-encoding transcriptomes. Proc Natl Acad Sci U S A 2004; 101: 6062-67.

34. Guleria I, Khosroshahi A, Ansari MJ, et al. A critical role for the programmed death ligand 1 in fetomaternal tolerance. J Exp Med 2005; 202: 231-7.

35. Munn DH, Mellor AL. IDO in the Tumor Microenvironment: Inflammation, Counter-regulation and Tolerance. Trends Immunol 2016; 37: 193-207.
36. Prendergast GC, Smith C, Thomas S, Mandik-Nayak L, Laury-Kleintop L, Metz R, Muller AJ. Indoleamine 2,3-dioxygenase pathways of pathgenic inflammation and immune escape in cancer. Cancer Immunol Immunother 2014; 63: 721-35.

37. Munn DH, Shafizadeh E, Attwood JT, Bondarev I, Pashine A, Mellor AL. Inhibition of T Cell Proliferation by Macrophage Tryptophan Catabolism. J Exp Med 1999; 189: 1363-72.

38. Wang J, Yu L, Jiang C, et al. Cerebral ischemia increases bone marrow CD4+CD25+FoxP3+ regulatory $T$ cells in mice via signals from sympathetic nervous system. Brain Behav Immun 2015; 43: 17283.

39. Perez R, Riese M, Lewis K, et al. Epacadostat plus nivolumab in patients with advanced solid tumors: Preliminary phase I/II results of ECHO-204. ASCO 2017 Meet. Abstr.

40. Sheppard KA, Fitz LJ, Lee JM et al. PD-1 inhibits T-cell receptor induced phosphorylation of the ZAP70/CD3zeta signalosome and downstream signaling to PKCtheta. FEBS Lett 2004; 574: 37-41.

41. Munn DH, Zhou M, Attwood JT et al. Prevention of allogeneic fetal rejection by tryptophan catabolism. Science 1998; 281: 1191-3.

42. Uyttenhove C, Pilotte L, Théate I et al. Evidence for a tumoral immune resistance mechanism based on tryptophan degradation by indoleamine 2,3-dioxygenase. Nat Med 2003; 9: 1269-74.

43. Huard B, Prigent P, Tournier M, Bruniquel D, Triebel F. CD4/major histocompatibility complex class II interaction analyzed with CD4and lymphocyte activation gene-3 (LAG-3)-Ig fusion proteins. Eur J Immunol 1995; 25: 2718-21.

44. Leitner J, Klauser C, Pickl WF, et al. B7-H3 is a potent inhibitor of human T-cell activation: No evidence for B7-H3 and TREML2 interaction. Eur J Immunol 2009; 39: 1754-64.

45. Ronchetti S, Zollo O, Bruscoli S, et al. GITR, a member of the TNF receptor superfamily, is costimulatory to mouse $T$ lymphocyte subpopulations. Eur J Immunol 2004; 34: 613-22.

46. Anderson AC. Tim-3: an emerging target in the cancer immunotherapy landscape. Cancer Immunol Res 2014; 2: 393-8.

\section{Address for correspondence}

\section{Paweł Tetrycz}

Department of Soft Tissue/Bone Sarcoma and Melanoma Maria Sklodowska-Curie Institute - Oncology Center

Roentgena 5

Warsaw, Poland

e-mail: pteterycz@coi.pl 